\title{
Metastatic Infiltrating Bladder Urothelial Carcinoma, Plasmacytoid Variant
}

National Cancer Institute

\section{Source}

National Cancer Institute. Metastatic Infiltrating Bladder Urothelial Carcinoma,

Plasmacytoid Variant. NCI Thesaurus. Code C157751.

Bladder plasmacytoid urothelial carcinoma that has spread to another anatomical site. 